\title{
Letter to the Editor Regarding "Prevalence of Intracranial Aneurysm in Patients with Aortopathy: A Systematic Review with Meta-Analyses"
}

\author{
Ajay Malhotra, Xiao Wu, Mihir Khunte \\ Department of Radiology and Biomedical Imaging, Yale University School of Medicine, New Haven, CT, USA
}

\section{Dear Sir:}

We would like to commend the authors Yu et al. ${ }^{1}$ for their study assessing the prevalence of intracranial aneurysms (IAs) in patients with aortopathy. Their review of the literature revealed a $12 \%$ prevalence of IAs in patients with aortopathy, leading them to conclude that screening for IA should be considered in patients with aortic disease.

One of the studies cited is Kuzmik et al. ${ }^{2}$ which reported a $9 \%$ prevalence of IA in patients with thoracic aortic aneurysms (TAA), and ran from 1997 to 2009. We recently published the results of a 9-year (2009 to 2018) prospective, follow-up screening study from the same institution, with a much larger sample size. Our study found the prevalence to be much smaller (4.9\%), and most of the aneurysms were small in size. ${ }^{3}$ The prevalence of IA was also higher in TAAs in the descending aorta (9.09\%), compared to the ascending aorta (4.51\%), and they are likely caused by different disease processes. While descending TAAs are almost invariably heavily calcified, thrombus containing, and closely related to traditional arteriosclerotic risk factors, ascending TAAs are rarely calcified, almost never contain thrombus, and are genetically mediated. Hypertension, smoking and female gender are known high risk predictors for $I A$, and it not clear from the study by Yu et al.' if these factors increase risk in patients with aortopathy, relative to the population without aortopathy. As regards genetic linkage, the data is heterogeneous. In a mega-analysis of a 1,000 Genomes Project-imputed, genome-wide association study, using data of four previously published aneurysm cohorts, van 't Hof et al. ${ }^{4}$ found that two established risk loci for IAs and TAAs had nominally significant effects; however, there was no evidence for polygenic overlap.

The rationale for screening high-risk populations is based on the $3.2 \%$ prevalence found in a standard 50-year-old man without any risk factors as reported in a 2011 meta-analysis. However, recent studies have shown a much higher prevalence due to the increased availability of cross-sectional imaging, with a population-based study showing a prevalence of 7\% in adults aged 35 to 75 years. ${ }^{5}$ Unruptured intracranial aneurysms (UIA) were found in $11.4 \%$ of patients who were imaged for acute stroke in a more recent study. ${ }^{6}$ If the prevalence is indeed as high as reported in these studies, it raises serious questions about previous recommendations of screening high risk populations, as their reported higher prevalence was only based on more aggressive screening in selected populations.

Screening recommendations should also take into account the possible growth and rupture risk of incidental aneurysms detected on these cross-sectional studies. Most are small aneurysms with low rates of growth and rupture, but they carry significant implications for downstream costs and patient outcomes. $^{7-9}$ There is significant heterogeneity in clinical practice regarding imaging surveillance of UIAs. ${ }^{10}$ Frequent imaging surveillance of small, incidentally-detected aneurysms indefinitely using magnetic resonance angiography does not result in better health outcomes, given the costs and complications associated with aneurysm ablation. ${ }^{8}$ Computed tomography angiography (CTA) is widely available, cheaper and has high spatial resolution, but it carries concerns of radiation exposure on prolonged surveillance. However, the radiation-induced brain cancer risk is likely low when compared to aneurysm rupture risk, and it does not significantly impact the cost-effectiveness of CTA surveillance of small, incidental aneurysms. ${ }^{9,11}$ There is, 
unfortunately, little literature available that establish if patients with aortopathy have an increased risk for aneurysm development and growth as compared to the normal population, since most studies have focused only on prevalence. Finding an incidental IA is also likely to add to patient stress and anxiety, and this should be taken into consideration when counseling patients to undergo imaging studies to look for asymptomatic, unruptured aneurysms.

\section{References}

1. Yu X, Xia L, Jiang Q, Wei Y, Wei X, Cao S. Prevalence of intracranial aneurysm in patients with aortopathy: a systematic review with meta-analyses. J Stroke 2020;22:7686.

2. Kuzmik GA, Feldman $M$, Tranquilli $M$, Rizzo JA, Johnson $M$, Elefteriades JA. Concurrent intracranial and thoracic aortic aneurysms. Am J Cardiol 2010;105:417-420.

3. Malhotra A, Seifert $K$, Wu X, Matouk $C$, Elefteriades JA. Screening for intracranial aneurysms in patients with thoracic aortic aneurysms. Cerebrovasc Dis 2019;47:253259.

4. van 't Hof FN, Ruigrok YM, Lee $\mathrm{CH}$, Ripke $\mathrm{S}$, Anderson $\mathrm{G}$, de Andrade $M$, et al. Shared genetic risk factors of intracranial, abdominal, and thoracic aneurysms. J Am Heart Assoc 2016;5:e002603.

5. Li MH, Chen SW, Li YD, Chen YC, Cheng YS, Hu DJ, et al. Prevalence of unruptured cerebral aneurysms in Chinese adults aged 35 to 75 years: a cross-sectional study. Ann Intern Med 2013;159:514-521.

6. Chen ML, Gupta A, Chatterjee A, Khazanova D, Dou E, Patel $\mathrm{H}_{1}$ et al. Association between unruptured intracranial aneurysms and downstream stroke. Stroke 2018;49:2029-2033.
7. Malhotra A, Wu X, Forman HP, Grossetta Nardini HK, Matouk $C C$, Gandhi D, et al. Growth and rupture risk of small unruptured intracranial aneurysms: a systematic review. Ann Intern Med 2017;167:26-33.

8. Malhotra A, Wu X, Forman HP, Matouk CC, Gandhi D, Sanelli P. Management of tiny unruptured intracranial aneurysms: a comparative effectiveness analysis. JAMA Neurol 2018;75:27-34.

9. Malhotra A, Wu X, Chugh A, Mustafa A, Matouk CC, Gandhi $D$, et al. Risk of radiation-induced cancer from computed tomography angiography use in imaging surveillance for unruptured cerebral aneurysms. Stroke 2019;50:76-82.

10. Malhotra A, Wu X, Geng B, Hersey D, Gandhi D, Sanelli P. Management of small unruptured intracranial aneurysms: a survey of neuroradiologists. AJNR Am J Neuroradiol 2018;39:875-880.

11. Wu X, Matouk CC, Mangla R, Forman HP, Gandhi D, Sanelli $P$, et al. Cost-effectiveness of computed tomography angiography in management of tiny unruptured intracranial aneurysms in the United States. Stroke 2019;50:23962403.

\footnotetext{
Correspondence: Ajay Malhotra

Department of Radiology and Biomedical Imaging, Yale University School of Medicine, Box 208042, Tompkins East 2, 333 Cedar St, New Haven, CT 06520-8042, USA

Tel: $+1-734-255-4799$

Fax: +1-203-785-3024

E-mail: ajay.malhotra@yale.edu

https://orcid.org/0000-0001-9223-6640

Received: May 30, 2020

Revised: May 30, 2020

Accepted: July 10, 2020

The authors have no financial conflicts of interest.
} 